\title{
MAMMARY-TYPE MYOFIBROBLASTOMAS: A REPORT ON FOUR CASES WITH EMPHASIS ON MOLECULAR PROFILING AND LITERATURE REVIEW
}

\author{
Anca EVSEI ${ }^{1}$, Javier F. ANDREU ${ }^{2}$, Carmen M. BLAZQUEZ MAÑA ${ }^{2}$, \\ Inmaculada M. MENDEZ COCA ${ }^{2}$, Ruben CARRERA SALA ${ }^{2}$, Jose A. VAZQUEZ LUQUE${ }^{2}$, \\ Carmen M. RAMOS GUIJO2 ${ }^{2}$, Rosa M. RODRIGUEZ MILLAN ${ }^{2}$, Mircea GHEORGHE ${ }^{1}$, \\ Adelina BIRCEANU-COROBEA ${ }^{1}$, Narcis COPCA $^{1}$ \\ ${ }^{1}$ Cesito Research Center, "Sf. Maria“ Clinical Hospital, Bucharest, Romania \\ ${ }^{2}$ Pathology Department, Hospital Parc Tauli, Sabadell, Barcelona, Spain
}

Received 29 Nov 2019, Accepted 04 Febr 2020

hitps://doi.org/10.31688/ABMU.2020.55.1.18

\begin{abstract}
Introduction. Myofibroblastomas of the breast are rare mesenchymal lesions thought to be derived from a CD34-positive mammary stromal cell that shows a great capacity for multipotency.

Cases presentation. We performed a retrospective analysis of 4 cases with confirmed mammary myofibroblastomas. Hematoxylin and eosin slides and immunohistochemical slides were reviewed. For next-generation sequencing studies we used a TruSight Tumor 15 panel which ran in a MiSeq sequencer and was analyzed with illumina VariantStudio 3.0 and IGV (Integrative Genomic Variation). The case series consisted of $4 \mathrm{pa}-$ tients ( 3 men, 1 woman). The anatomic location distribution was equal, with a 1:1 ratio between the left and right breast and a gross median size of the lesion of $34 \mathrm{~mm}$. Gross features revealed a well circumscribed lesion with a vaguely lobular cut surface and the microscopical features were that of a classic-type MFB composed of benign, spindle-shaped cells interrupted
\end{abstract}

\section{Résumé}

Myofibroblastomes de type mammaire: rapport sur quatre cas avec accent sur le profil moléculaire et revue de la littérature

Introduction. Les myofibroblastomes (MFB) du sein sont des lésions mésenchymateuses rares, supposées d'être dérivées d'une cellule stromale mammaire CD34-positive, présentant une grande capacité de multipotence.

Rapport du cas. Nous avons mené une étude rétrospective sur 4 cas de myofibroblastomes mammaires confirmés entre 2003 et 2018. Des lames d'hématoxyline et d'éosine et des lames immunohistochimiques ont été passées en revue. Pour les études de séquençage de nouvelle génération, nous avons utilisé un panneau TruSight Tumor 15, qui fonctionnait dans un séquenceur MiSeq et qui a été analysé avec illumina Variant Studio 3.0 et VGI (Variation génomique intégrative). La série de cas comprenait 4 patients avec un rapport 
by thick collagen fibers that show extensive hyalinization. CD34 and desmin immunohistochemical markers were diffusely positive in tumor cells, thus confirming the diagnosis. Next generation sequencing used a TruSight Panel 15 and revealed mutations in TP53, p.P72R gene in two cases and in ERBB2 p.1655V, KIT c.2484C>T, GUSB p.V270M genes in one case. No pathogenic mutations were identified.

Conclusions. The diagnosis of these tumors is challenging based on the rareness of these lesions. Molecular analyses are important to confirm the diagnosis based on morphology and immunohistochemistry. Given the fact that these tumors are rare and benign, there is little data about the molecular signature of this tumor.

Keywords: breast, myofibroblastoma, next generation sequencing. hommes / femmes de 3: 1. La distribution de la localisation anatomique était égale avec un rapport 1: 1 entre les seins gauche et droit et une taille médiane brute de la lésion de $34 \mathrm{~mm}$. Les caractéristiques globales révélaient une lésion bien circonscrite avec une surface de coupe vaguement lobulaire. Les caractéristiques microscopiques étaient celles d'un MFB de type classique composé de cellules bénignes en forme de fuseau, interrompues par des fibres de collagène épaisses, présentant une hyalite étendue. Les marqueurs immunohistochimiques CD34 et desmine étaient positifs de manière diffuse dans les cellules tumorales, confirmant ainsi le diagnostic. Le séquençage de nouvelle génération a utilisé un panel 15 de TruSight et a révélé des mutations dans les gènes TP53, p.P72R et dans les gènes ERBB2 p.1655V, KIT c.2484C> T, GUSB p.V270M dans un cas. Aucune mutation pathogène n'été pas identifiée.

Conclusions. Le diagnostic de ces tumeurs est difficile en raison de la rareté de ces lésions. Les analyses moléculaires sont importantes pour confirmer le diagnostic donné, basé sur la morphologie et l'immunohistochimie avec une méthode indépendante pour fournir des informations sur le pronostic. Étant donné que ces tumeurs sont rares et bénignes, peu de recherches ont été effectuées sur la signature moléculaire de cette tumeur.

Mots-clés: sein, myofibroblastome, séquençage de prochaine génération.

have found an almost equal distribution between the male and postmenopausal female population ${ }^{5,6}$. Age distribution is very wide, with a range varying from 35 to 85 years old? Clinical presentation usually consists of a patient complaining of a firm, mobile, solid breast nodule. Mammographic views usually show a round to oval, homogenous, lobulated tumor without dystrophic intra tumoral calcifications; this aspect can be easily confused with a fibroadenoma or hamartoma of the breast ${ }^{8}$. Microscopically, this entity is composed of benign-looking, fusiform cells with myofibroblast differentiation; the stroma is usually hyalinized and associates frequent mast cells or a fatty component. Unusual morphological features have been described by several authors, but there is no relevant connection to an aggressive clinical behavior ${ }^{9}$. Immunohistochemically, CD34 and desmin positivity usually confirms the diagnosis. Genetically, MFB is part of the so-called $13 \mathrm{q} / \mathrm{Rb}$ family of tumors which possess deletion or rearrangement of $13 \mathrm{q} 14$, usually confirmed by molecular testing such as FISH $^{10}$ and resulting in loss of $\mathrm{Rb}$ positivity using immunohistochemical testing ${ }^{11}$. Differential diagnosis is of the 
utmost importance in this case because of the rarity and the wide spectrum of pathological patterns. Subsequently, this tumor can easily be mistaken for other benign and malignant breast entities, with fusiform cells causing real diagnostic difficulties ${ }^{12}$. Next-generation sequencing (NGS) technology with its high-throughput capacity and low cost has developed rapidly in recent years and has become an important analytical tool in genomic research. This powerful technology has the ability to detect thousands or even hundreds of thousands of genetic variants in a single test run ${ }^{13}$. Considering the unclear molecular background of MFBs, our purpose was to shed some light on the genetic alterations and build a molecular profile that can further offer insight into benign spindle cell lesions of the breast.

\section{Cases presentation}

This study was approved by the institution ethics committee. All cases $(n=4)$ diagnosed as myofibroblastomas of the breast between 2003 and 2018 were retrieved from the electronic medical database of the Pathology Department of Hospital Parc Tauli, Sabadell, Spain. Representative hematoxylin and eosin stained slides and immunohistochemically stained slides were reviewed and the diagnosis of MFB was confirmed. We took into consideration the patients' age and sex, anatomic location of the tumor, size of tumor (maximal dimension), type of sampling (biopsy versus excision) and margin status when possible. An automatically (DAKO autostainer or ROCHE Ventana) immunohistochemistry was performed for desmin, SMA, BCL2, ER, PR and Ki67.

Next Generation sequencing standard protocols comprised the use of the TruSight Tumor 15 panel in all cases. The specimens were run in a MiSeq sequencer and analyzed with Illumina VariantStudio 3.0 and IGV (Integrative Genomic Variation). Filters used as a control were $\times 500$ minimum read depth and $>5 \%$ frequency of the variants. We didn't annotate polymorphisms in intronic, 5'UTR, 3'UTR regions or synonymous mutations.

The case series consisted of 4 subjects and included one woman and three men. The mean age at diagnosis was 68 years, with a wide overall range (44 to 97 years). The anatomic distribution was equal, with a 1:1 ratio between the left and right breast, with one case in the retro-areolar region and one case located in the superior quadrant. Radiological findings revealed a heterogeneous, well-circumscribed mass on mammography and a hyperechoic, solid tumor with increased vascularity on ultrasound (Figures 1A,1B,1C). The pathological specimen was

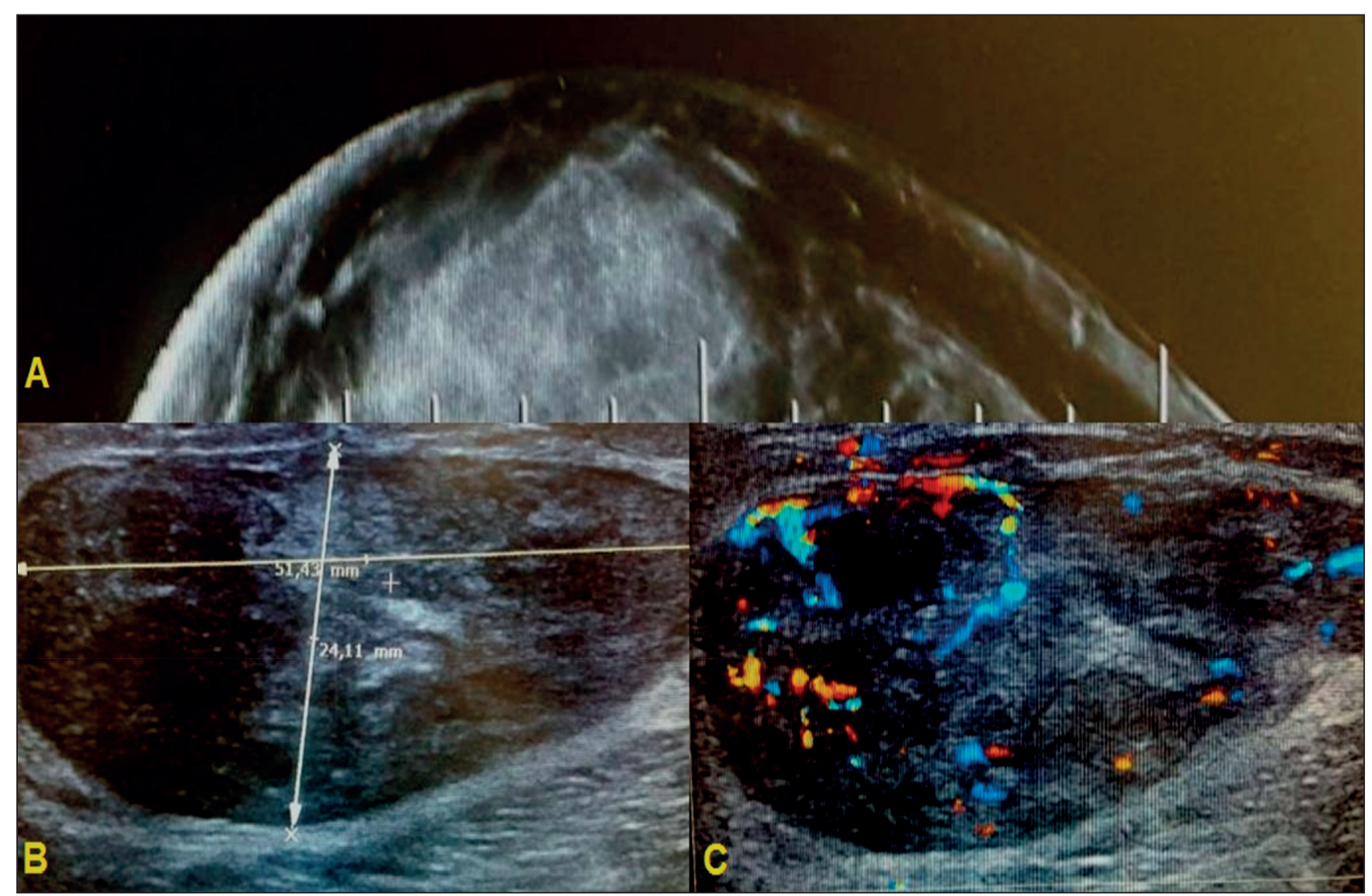

Figure 1. Myofibroblastoma of the breast: A. Mammography: Circumscribed, high-density mass; B, C: Ultrasound: Hyperechoic, well-defined mass with increased vascularity (Doppler). 


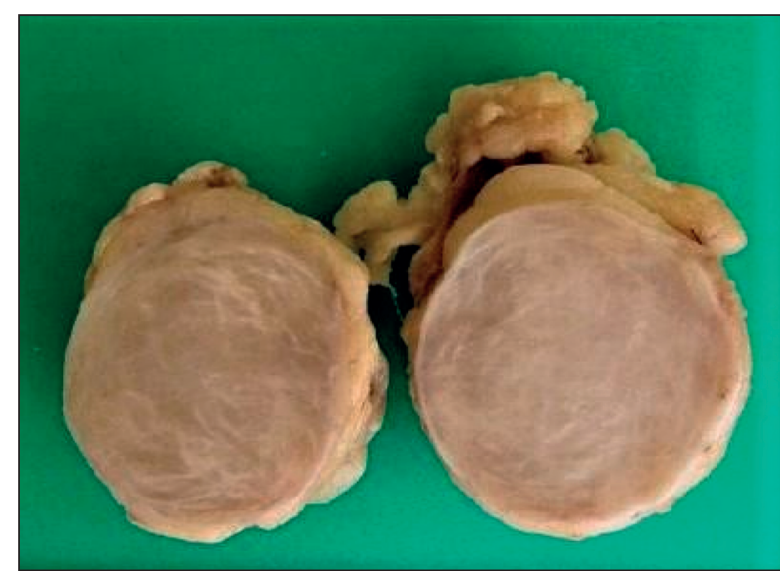

Figure 2. Gross features revealed a well-circumscribed lesion with a vaguely lobular cut surface.

documented in all patients. Three cases had breast core needle biopsies followed by tumor excision and only one was diagnosed using fine needle aspiration and subsequent core needle biopsy. Grossly, the tumors were generally described with a rubbery to gelatinous and yellow to white /gray cut surface (Figure 2). The macroscopic tumor size was documented in three out of four cases, with a mean of $34 \mathrm{~mm}$ (range 1 to $60 \mathrm{~mm}$ ). One case out of three was documented to have focal positive resection margins, but the patient did not undergo surgical re-excision.

Slides were available for review for all four cases. Microscopically, myofibroblastoma is composed of short fascicles of uniform, bipolar, spindle-shaped cells between bands of hyalinized, broad collagen with frequent mast cells resembling spindle cell lipomas (Figure 3.A). No myxoid, adipose, leiomyomatous or chondroid changes were noticed in the stroma of the four cases. The fusiform cells showed round nuclei with irregular nuclear contours and dispersed chromatin with distinct nucleoli (Figure 3.B). Little nuclear pleomorphism was recorded with mitotic figures generally less than 2 per 10 high-power fields. No unusual morphological patterns (epithelioid, highly cellular, with cytological atypia etc.) were identified.

Immunohistochemically, all cases were CD34 and desmin positive, with most cases showing multifocal and diffuse staining (Figure 4.A, 4.B). Vimentin (Figure 4.C) was usually diffusely positive in all cases. Additional stains included 2 cases with bcl2 positivity and 1 case with ER and PR nuclear positivity. SMA and S100 (Figure 4.D, 4.E) were usually negative and in some cases weakly positive. Follow up data was limited because of the benign nature of this lesion.

Next generation sequencing used a TruSight Panel 15 and revealed mutations in TP53, p.P72R gene in two cases and in ERBB2 p.1655V, KIT
c.2484C>T, GUSB p.V270M genes in one case. No pathogenic mutations were identified.

Myofibroblastomas of the breast are rare tumors with myofibroblast differentiation and are part of a group of CD-34 related tumors (for example, spindle cell lipoma or cellular angiofibroma) ${ }^{1}$. The tumor cells were regarded as myofibroblasts based on ultrastructural studies as the tumor cells showed cytoplasmic stress fibers, cell junctions and basal-like material that fulfilled the criteria used for defining these cells $s^{4}$. Magro et al stipulated in their study that the point of origin of these tumors is a precursor of mesenchymal origin that shows overlapping histological and immunohistochemical features ${ }^{13}$. The CD34 positive mammary stromal cell is believed to play an important role in the histogenesis, considering its ability to differentiate along several mesenchymal lines - fibroblast, myofibroblast, adipocytic, osseous, cartilaginous, leiomyomatous etc ${ }^{14}$. This cellular potential could explain the association of different types of cells (fibroblasts, myofibroblasts, adipocytes etc), including some heterologous elements (smooth muscle, osseous, cartilaginous) in the same $\mathrm{MFB}^{15}$. It is of the utmost importance to get an insight in the molecular background to determine which factors control growth and myofibroblast proliferation ${ }^{16}$. The mechanism of growth is believed to be influenced by cytokine secretion, both in an autocrine and paracrine way. Particular examples are TGFbeta, TNF and fibroblast peptide ${ }^{17}$. Another mechanism of growth has been attributed to hormone stimulation, in part based on estrogen and progesterone expression and on the strong association in literature to gynecomastia, PASH or hormonal treatment ${ }^{17-19}$. One author reports the first case of a rare MFB in a transgender patient receiving estrogen therapy, a unique case in the setting of gender-affirming hormone therapy $y^{20}$.

The first 16 cases published in literature ${ }^{3}$ outlined a male predominance for this lesion, but subsequent reports have established an equal distribution in postmenopausal women and older men ${ }^{5}$, probably because of a higher incidence of mammography. In our series, it was described more often in older men, thus confirming the earlier reports. The age at presentation is very wide, ranging from 40-87 years old. Clinically, this type of tumor presents as a solitary, painless, firm, mobile mass, usually unilateral, which grows slowly for a long period of time ${ }^{21}$. Nonetheless, there have been reported cases with giant tumors, with massive enlargement of the breast and associated pain, clinically suspected to be phyllodes tumor ${ }^{22}$. Local excision is usually curative, with no evidence of recurrence or distant metastasis.

Breast myofibroblastomas have been described in various case reports, with a versatile radiological 


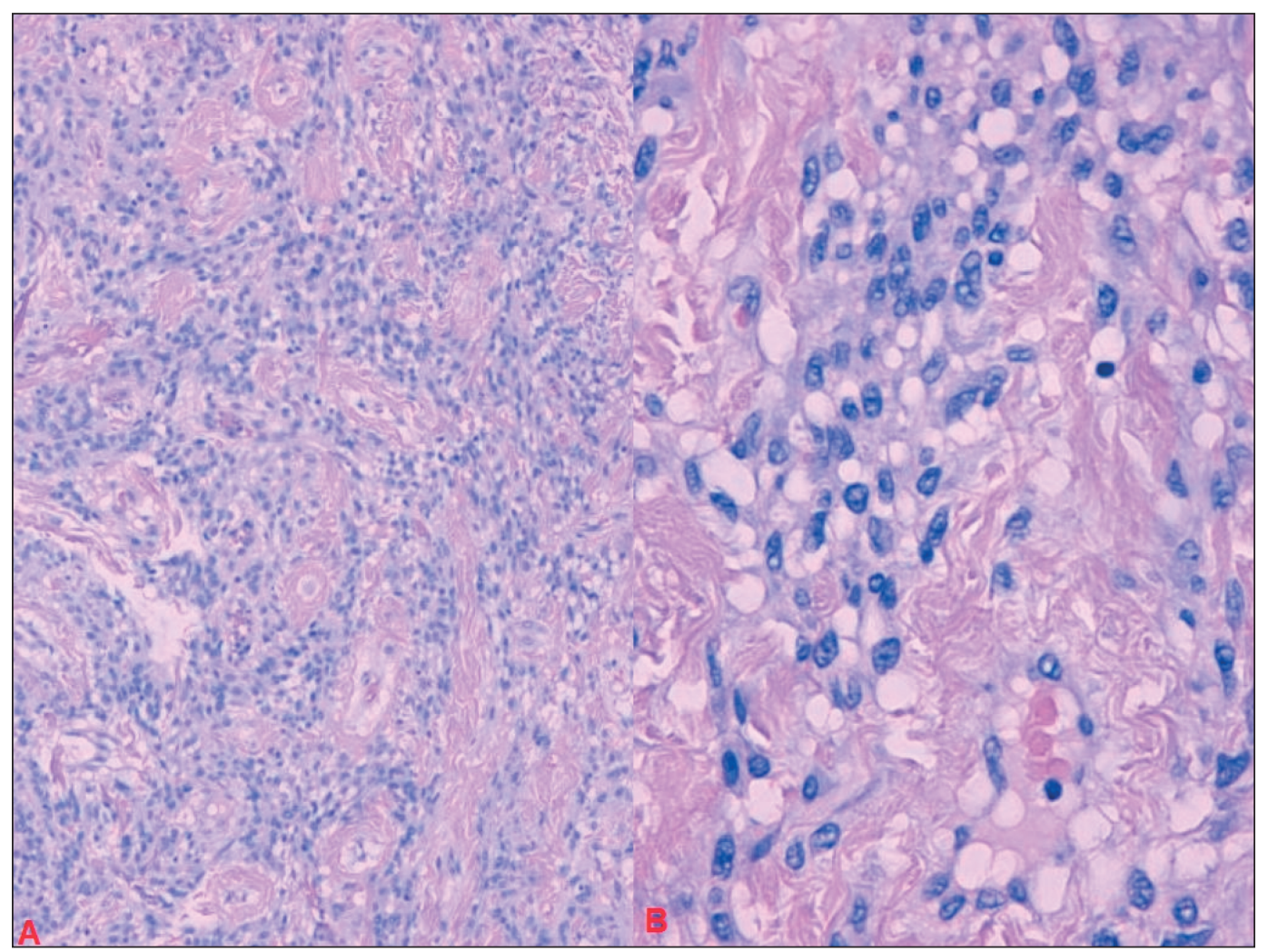

Figure 3. Myofibroblastoma of the breast, classic type: A: Ob 10x: The tumor is composed of a homogenous population of predominantly spindle-shaped cells with ovoid nuclei and pale cytoplasm, arranged in intersecting bundles. B: Ob 40x: The tumor shows frequent collagen bands among the tumor cells.

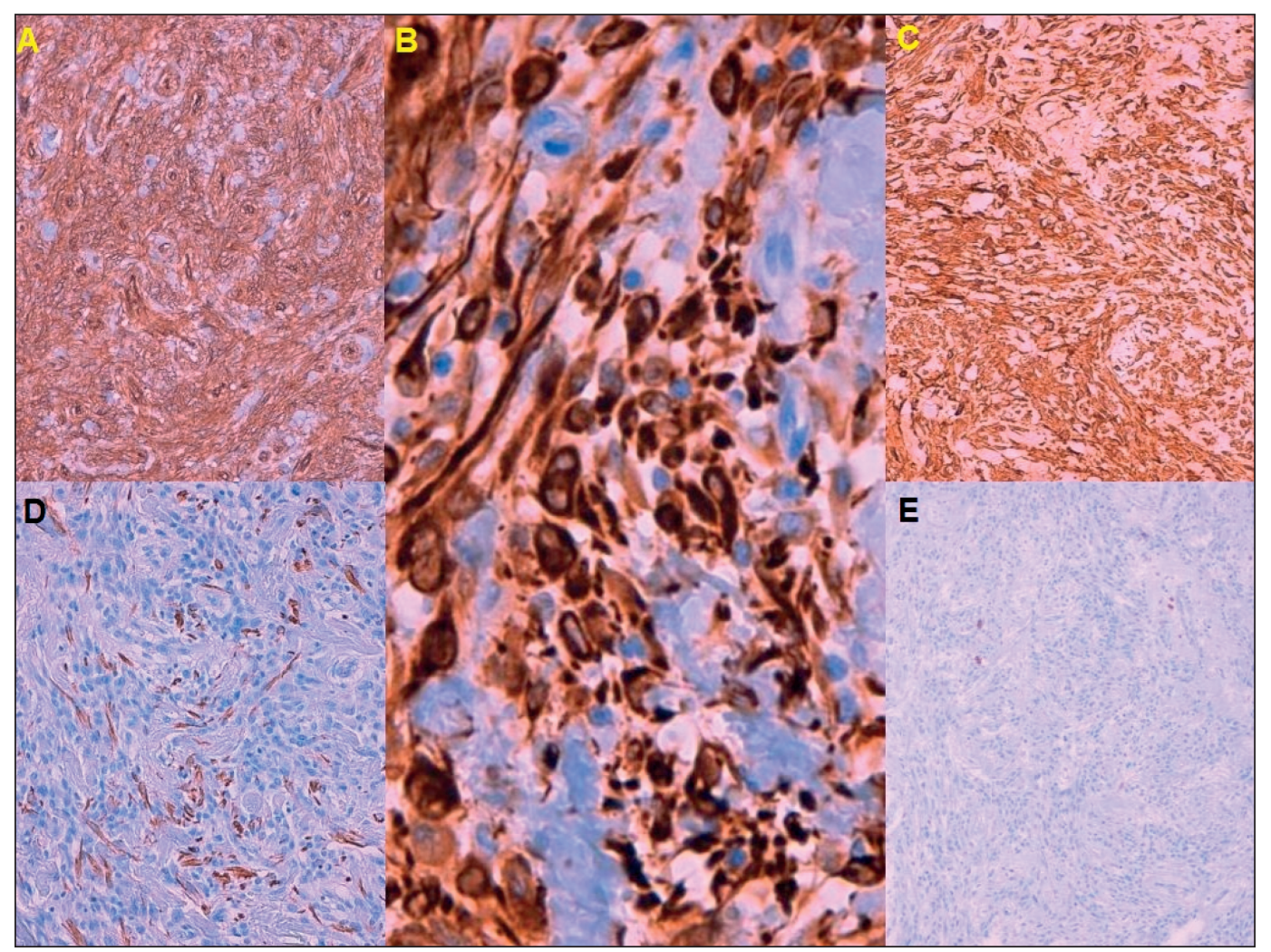

Figure 4. A: Ob 10x: Intense immunoreactivity for CD34 in tumor cells; B: Ob 40x: Intense immunoreactivity for Desmin in tumor cells; C: Ob 20x: Intense immunoreactivity for Vimentin in tumor cells; D: Ob 20x: SMA negative in tumor cells; E: Ob 10x: S100 negative in tumor cells. 
presentation, nonspecific. Mammography depicts in most cases a circumscribed, round to oval mass, with variable density ${ }^{23}$. They may show micro lobulated margins and usually do not associate microcalfications $^{24}$. Sonographic findings usually vary from a homogenous hypoechoic pattern to a more heterogeneous one, with or without posterior shadowing ${ }^{25}$. In female patients, it may exist an overlap in sonographic findings, as the MFB pattern of a circumscribed mass with variable echogenicity may raise the question of a fibroadenoma. Doppler ultrasonography may show increased vascularity. There is little information in the literature about magnetic resonance (MR) findings in this lesion. Some studies have described MFB showing a hyperintense signal on T2-weighted image $^{26}$. Our findings on Doppler's sonography usually showed a solid tumor mass with diffuse iso-echogenicity, circumscribed margins, and increased vascularization, which is consistent with literature data. Although mammographic and sonography of MFBs are not pathognomonic, their imaging appearance is definitely unusual comparing to the typical image of breast cancer. That is why the differential diagnosis of tumors in the male breast should be very thorough.

Although it is relatively easy to diagnose a typical $\mathrm{MFB}$ on a resection specimen, fine-needle aspiration cytology (FNAC) remains ambiguous in some cases, often leading to a misdiagnosis, such as gynecomastia or phyllodes tumor ${ }^{27}$. The aspirates usually show randomly arranged, single and/ or clusters oval to spindle-shaped cells, fine cytoplasm, with occasional nuclear pleomorphism with irregular nuclear membranes and nuclear pseudo-inclusions; collagenous extracellular matrix may be identified ${ }^{28}$. In addition, if the pathologist is confronted with unusual morphologic variants showing atypical characteristics, the diagnosis becomes more difficult. From a cytodiagnostic point of view, there are only 21 cases of MFB with cytological evaluation by FNAC reported in the literature. One study set out to correlate findings on FNAC and cell block material, both morphologically and immunohistochemically, and concluded that these tests complement each other ${ }^{28}$.

The average lesion dimension is about $2 \mathrm{~cm}$, but it can vary from a few millimetres to over $20 \mathrm{~cm}$. Gross examination usually reveals a well circumscribed lesion, with a vaguely lobular cut surface, homogenous, bulging grey to pink which rarely shows myxoid changes and almost never necrosis, hemorrhage or cystic degeneration ${ }^{29}$. In some cases, it may show extensive lipomatous areas. Our cases fit the general characteristics for gross examination.

From a histological point of view ${ }^{4,27,30}$, the classic-type of MFB is a well-circumscribed benign-looking, fusiform, spindle-shaped cells closely packed in short, straight, haphazardly intersecting fascicles or clusters of cohesive cells, interrupted by hyalinized collagen bundles with prominent stromal mast cells; the nuclei of the spindle cells are round to stubby or oval with irregular nuclear contours and dispersed chromatin with distinct nucleoli, with little pleomorphism and low mitotic count (0-2 mitoses per 10 high-power fields); occasionally, the nuclei may have grooves or pseudo-inclusions. In some areas, tumor cells may show palisading of nuclei. Apart from the classic type of $\mathrm{MFB}$, this rare lesion can be identified under several unusual histological variants. The cellular pattern of MFB exhibits a highly cellular tumor ${ }^{28}$; the epithelioid variant of MFB is defined by tumors composed predominantly (>50\%) of epithelioid cells $\mathrm{s}^{27,30}$. Because of this wide range of appearances, this variant is susceptible to misdiagnosis, especially with an invasive carcinoma. One study reports an epithelioid type MFB mimicking an invasive lobular carcinoma, with a microscopic tableau composed of epithelioid cells dispersed in a fibro-fatty stroma and a pseudo infiltrative growth pattern ${ }^{30}$. One uncommon variant is the myxoid type of MFB characterized by myofibroblast proliferation embedded in a predominantly myxoid stroma ${ }^{2,15}$. The leiomyomatous $\mathrm{MFB}$ is very unusual, resembling leiomyomas of Mullerian-type and poses a great diagnostic challenge. The lipomatous variant is represented by a tumor with stroma composed of predominantly $75 \%$ adipose tissue. This particular variant is very important to be recognized as it may often be mistaken for benign or malignant spindle cell tumors, such as spindle cell lipoma or low-grade liposarcoma ${ }^{20,22}$. The infiltrating variant is characterized by an invasive growth pattern with entrapment of fat, mammary ducts and lobules mimicking fibromatosis ${ }^{26}$. Decidual-like MFB is characterized by a very rare architectural pattern composed of large cells which grow in a solid, trabecular or nested architectural pattern with abundant, eosinophilic cytoplasm and sharp cellular borders ${ }^{16,17}$. Only one case was described in the literature so far, by Magro et al. Immunohistochemical studies and differential diagnosis are crucial for ruling out malignancy in this case.

In addition to the unusual variants of $\mathrm{MFB}$, there have been reports which described curious morphologic features ${ }^{2}$. Atypical, bizarre mono- or multinucleated cells with variable degrees of nuclear pleomorphism have been reported in different variants of MFB, but their nature is believed to be degenerative, similar to other soft tissue tumors. Multinucleated floret-like cells have been described in epithelioid-type MFB and they usually resemble the cells commonly present in pleomorphic lipoma. MFB can also have areas of heterologous differentiation, mostly adipose tissue and rarely leiomyomatous, 
osseous or cartilaginous. Occasionally, one may find MFB with haemangiopericytoma-like pattern, which is a rather common architectural pattern present in several soft tissue tumors ${ }^{2,3}$.

Most MFB are immunohistochemically SMA, calponin and desmin positive, fact that supports the myofibroblast origin of these tumors ${ }^{1}$. Some cases showed focal expression of h-caldesmon in scattered cells, a fact that suggests that mammary fibroblasts are capable of undergoing smooth muscle differentiation $^{12}$; MFB are usually positive for ER, PR and AR. CD34, CD99 and bcl2 are frequently positive whereas keratins are negative ${ }^{1}$. However, D'Alfonso et al describe a subset of MFB with absent or focal expression of CD34, an uncommon occurrence in an already rarely encountered breast tumor ${ }^{30}$. Other studies reported this unusual feature in MFB with leiomyomatous differentiation ${ }^{28,29}$.

Cytogenetic studies have proven chromosome 13 rearrangements associated with the loss of the $13 \mathrm{q} 14$ chromosomal region and partial loss of $16 \mathrm{q}$ in mammary MFB ${ }^{27}$. This characteristic usually tested using FISH which assesses RB1 loss, links MFB to spindle cell lipomas and cellular angiofibromas on a genetic level ${ }^{1,10,30}$. Although there is an immunohistochemical equivalent for RB1 testing, some authors suggest that the correlation between immunohistochemistry and FISH is not reliable ${ }^{27}$.

Differential diagnosis for MFB is very broad and dependent on the many unusual morphological variants mentioned above ${ }^{11}$. The panel includes solitary fibrous tumor, spindle cell lipoma, smooth muscle and neural tumors, metaplastic carcinoma, myoepithelioma, fibromatosis, nodular fasciitis etc ${ }^{7,30}$. Immunohistochemical testing usually aids in differentiating between these entities.

Next-generation sequencing has gained a lot of momentum recently, especially because of its ability to sequence normal and cancer genome in matter of weeks. Massively parallel sequencing can be used for the characterization at the same time of cancer genomes in terms of somatic base pair and in-del mutations, balanced and unbalanced rearrangements, and copy number changes in a single experiment. Another important use for NGS consists in focused analysis of specific genomic regions, specific genes or the whole exome. In fact, the Breast Cancer International Cancer Genome Consortium has promised to complete sequencing the genome of 1,500 breast cancers. This study will focus on general alteration in the breast cancer in general and in different subtypes of breast cancer $^{23}$. Although myofibroblastomas are rare and benign lesions, their genetic background is not very well documented. The few mutations we found in our study showed no pathogenic characteristics, but nonetheless, to our knowledge, this is a first step towards uncovering the molecular features of these rare tumors.

Literature review revealed two cases treated with mastectomy after an incorrect malignant diagnosis and there have been a few cases in men with very large tumors that required this procedure. Nonetheless, the surgical treatment is represented by complete tumor excision with a wider intervention if the tumor is present at a margin ${ }^{28,29}$. The risk of recurrence for MFB is extremely low. However, there have been reports of two cases that recurred after 17 and, respectively 20 years ${ }^{11}$. Although MFB is a benign tumor with very low recurrence rate, one might take into consideration this tumor's risk for late recurrence.

\section{Conclusions}

The diagnosis of these tumors is challenging because of their rareness. Molecular analyses are important to confirm the diagnosis, based on morphology and immunohistochemistry with an independent method to provide information about prognosis. Given the fact that these tumors are rare and benign, there is little research done for the molecular signature of this tumor. To our knowledge, this is one of few attempts to get an insight of the genetic alterations of mammary myofibroblastomas.

\section{Author contributions}

J.A., C.B., and I.M. made the histopathological diagnosis, R.C., J.V., C.R., and R.R. did the immunohistochemical and molecular analysis. A.E., A.B. ,M.G., and N.C. wrote the manuscript. All authors have read and agreed to the published version of the manuscript.

\section{Compliance with Ethics Requirements:}

„The authors declare no conflict of interest regarding this article"

"The authors declare that all the procedures and experiments of this study respect the ethical standards in the Helsinki Declaration of 1975, as revised in 2008(5), as well as the national law. Informed consent was obtained from all the patients included in the study"

"No funding for this study"

\section{Acknowledgments: none}

\section{References}

1. Krings G, Mcintire P, Shin S. Myofibroblast, fibroblastic and myoid lesions of the breast. Seminars in Diagnostic Pathology. 2017;34: 427-437.

2. Magro G. Mammary myofibroblastoma: a tumor with a wide morphologic spectrum. Archives of Pathology $\mathcal{E}$ Laboratory Medicine. 2008;132(11):1813-1820. 
3. Wargotz ES, Weiss SW, Norris HJ. Myofibroblastoma of the breast. Sixteen cases of a distinctive benign mesenchymal tumor. Am J Surg Pathol. 1987; 11(7): 493-502.

4. Tavassoli FA, Eusebi V (eds). Benign soft tissue lesions, in Tumors of the Mammary Gland, AFIP Atlas of Tumor Pathology Series 4, Fascicle 10, Washington DC, American Registry of Pathology, 2009:265-269.

5. Magro G, Bisceglia M, Michal M. Expression of steroid hormone receptors, their regulated proteins, and bcl-2 protein in myofibroblastoma of the breast, Histopathology. 2000;36(6):515-21.

6. Soyeon A, Song JS, Park S, et al. Mammary type myofibroblastoma: a report of two cases Journal of Pathology and Translational Medicine. 2016;50: 385-389.

7. McMenamin ME, Fletcher CD. Mammary-type myofibroblastoma of soft tissue: a tumor closely related to spindle cell lipoma. Am J Surg Pathol. 2001;25(8):1022-9.

8. Raut P, Lillemoe TJ, Carlsn A. Myofibroblastoma of the breast. Appl Radiol. 2017; 46(3):42-44.

9. Zamecnik M, Macuch K. Mammary leiomyomatous-type myofibroblastoma with symplastic atypia. J Interdiscipl Histopathol. 2017; 5(3):103-105.

10. Uehara K, Ikehara F, Shibuya R, et al. Molecular signature of tumors with monoallelic 13q14 deletion: a case series of spindle cell lipoma and genetically-related tumors demonstrating a link between FOX01 status and p38 MAPK pathway. Pathol Oncol Res. 2018;24:861-869.

11. Howitt EB, Fletcher DMC. Mammary-type myofibroblastoma clinicopathologic characterization in a series of 143 cases. Am J Surg Pathol. 2016; 40:361-367.

12. Huang Y, Chen F. Review of myofibroblastoma of breast and its most common mimickers. NAJ Med Sci. 2012;5(1):38-42.

13. Park ST, Kim J. Trends in next-generation sequencing and a new era for whole genome sequencing. Int Neurourol J. 2016;20(2):76-83.

14. Magro G, Michal M, Bisceglia M. Benign spindle cell tumors of the mammary stroma: diagnostic criteria, classification, and histogenesis. Pathol Res Pract. 2001;197(7): 453-66.

15. Fukunaga M, Ushigome S. Myofibroblastoma of the breast with diverse differentiation. Arch Pathol Lab Med. 1997;121: 599-603.

16. Mele M, Jensen V, Wronecki A, et al. Myofibroblastoma of the breast: case report and literature review. International Journal Of Surgery Case Reports. 2011; 93-96.
17. Reis-Filho SJ, Faoro NL, Gasparetto LE, et al. Mammary epithelioid myofibroblastoma arising in bilateral gynecomastia: case report with immunohistochemical profile. Int J Surg Pathol. 2001;9(4): 331-4.

18. Solak A, Solak I, Genc B. Myofibroblastoma accompanied with bilateral gynecomastia. J Breast Health. 2013;9: 96-9.

19. Yoo, C, Pui J, Torosian M. Myofibroblastoma associated with bilateral gynecomastia: a case report and literature review. Oncology Reports. 1998; 5(3): 731-734.

20. O'Bryan J, Wolf-Gould C, Matsuo Y. Mammary myofibroblastoma in a transgender patient on feminizing hormones: literature review and case report. Transgender Healthwol. 2018;3(1): 1-9.

21. Metry M, Shaaban M, Youssef M, et al. Myofibroblastoma of the breast: Literature review and case report. Case reports in Oncological Medicine. 2016; 1714382.

22. Shah SN. Giant myofibroblastoma of breast: a case report. Indian J Pathol Microbiol. 2007; 50(3):583-5.

23. Comer DJ, Cui X, Eisen CS, et al. Myofibroblastoma of the male breast : a rare entity with radiologic - pathologic correlation. Clin Imaging. 2017; 42:109-112.

24. Yilmaz R, Akkavak G, Ozgur E, et al. Myofibroblastoma of the breast: ultrasonography, mammography and magnetic resonance imaging features with pathologic correlation. Ultrasound Quarterly. 2018;34(2):99-102.

25. Rochlis E, Germaine P. Radiologic presentation of a myofibroblastoma of the adult male breast. Radiology Case Reports. 2017;12(3):439-442.

26. Vourtsi A, Kehagias D, Antoniou, A et al. Male breast myofibroblastoma and MR findings. J Comput Assist Tomogr. 1999;23:414-416.

27. Magro G. Mammary myofibroblastoma. Arch Pathol Lab Med. 2008; 132:1813-1820.

28. Negri S, Bonzanini M, Togni R, et al. Fine needle aspiration of myofibroblastoma of the breast. Case report. Pathologica. 1995;87(6): 719-22

29. Fügen A, Elif S, Gülistan $G$, et al. Fine-needle aspiration cytology of a mammary myofibroblastoma: A case report on the role of immunohistochemistry and cell block preparations and a review of the literature. Diagn Cytopathol. 2016;44: 1064-1069.

30. Schmitt FC, AC Mera A. Fine needle aspiration cytology presentation of a cellular variant of breast myofibroblastoma. Report of a case with immunohistochemical studies. Acta Cytol. 1998; 42(3):721-4. 